
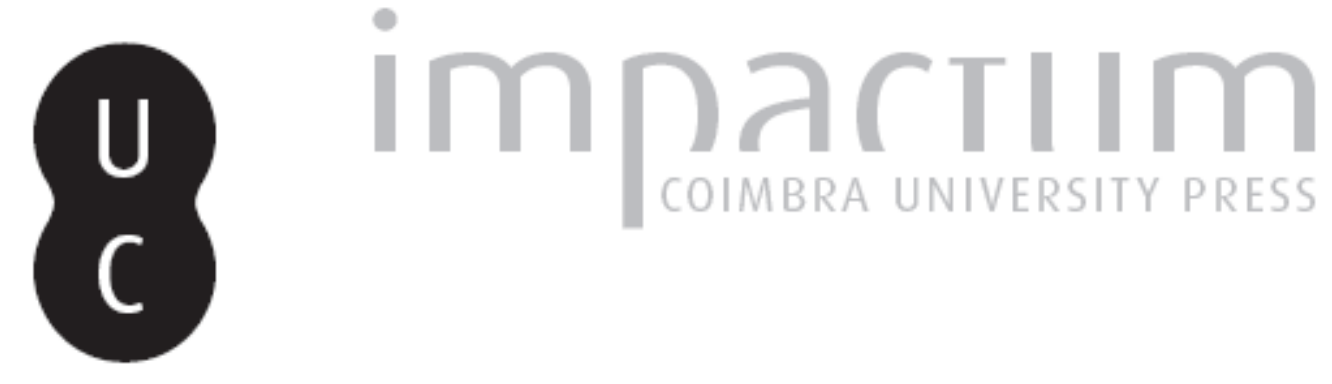

\title{
Utilização do cadastro territorial multifinalitário na gestão de riscos
}
Autor(es): $\quad$ Amorim, Amilton; Sousa, Ana Maria Rodrigues Monteiro de; Sousa, Eric Rafael Pereira de

Publicado por: Associação Portuguesa de Riscos, Prevenção e Segurança

URL persistente:

URI:http://hdl.handle.net/10316.2/36139

DOI:

DOI:http://dx.doi.org/10.14195/1647-7723_16_2

Accessed : $\quad$ 26-Apr-2023 15:11:40

A navegação consulta e descarregamento dos títulos inseridos nas Bibliotecas Digitais UC Digitalis, UC Pombalina e UC Impactum, pressupõem a aceitação plena e sem reservas dos Termos e Condições de Uso destas Bibliotecas Digitais, disponíveis em https://digitalis.uc.pt/pt-pt/termos.

Conforme exposto nos referidos Termos e Condições de Uso, o descarregamento de títulos de acesso restrito requer uma licença válida de autorização devendo o utilizador aceder ao(s) documento(s) a partir de um endereço de IP da instituição detentora da supramencionada licença.

Ao utilizador é apenas permitido o descarregamento para uso pessoal, pelo que o emprego do(s) título(s) descarregado(s) para outro fim, designadamente comercial, carece de autorização do respetivo autor ou editor da obra.

Na medida em que todas as obras da UC Digitalis se encontram protegidas pelo Código do Direito de Autor e Direitos Conexos e demais legislação aplicável, toda a cópia, parcial ou total, deste documento, nos casos em que é legalmente admitida, deverá conter ou fazer-se acompanhar por este aviso.

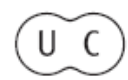




\section{territorium}

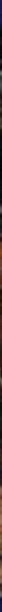

16

Revista da Associação Portuguesa de Riscos, Prevenção e Segurança 2009 
UTILIZAÇÃO DO CADASTRO TERRITORIAL MULTIFINALITÁRIO NA GESTÃO DE RISCOS*

\author{
Amilton Amorim \\ Universidade Estadual Paulista - UNESP, Presidente Prudente, SP, Brasil \\ amorim@fct.unesp.br
}

Ana Maria Rodrigues Monteiro de Sousa

Universidade do Porto - FLUP, Porto, Portugal

anamonteirosousa@gmail.com

Fric Rafael Pereira de Sousa

Universidade Estadual Paulista-UNESP, Presidente Prudente, SP, Brasil.

eusjc1987@hotmail.com

\title{
RESUMO
}

Num primeiro mamento, este trabalho apresenta uma breve revisão bibliográfica scbre cadastro e gestão territorial. Em seguida, é apresentada a metodologia utilizada para a execução de mapas temáticos pela Intemet, mostrandb as características de edificaçães susceptíveis a alguns tipos de riscos. Finalmente, evidenciase a potencialidade e a viabi lidade da uti 7 ização das informaçães cadastrais nesta importante área de aplicação, compoucos recursos financeiros.

Palavras chave: Cadastro Territorial Multifinalitário, SIG, gestão de riscos.

\section{RESUMÉ}

L'utilisation du cadastre multi-fonction dans la gestion du risque - ce travail présente dans un premier temps une brève revue bibliographique au sujet du cadastre et de la gestion du risque. Ensuite, la méthooblogie uti i isée dens la réalisation de cartes thématiques via Intemet montrant les caractéristiques des édifices potentiellement exposés au risque est présentée. Finalement, ce travail met en lumière le potentiel et la viabilité de ces informations, dotenues avec des ressarrces financières limitées, quant aux cadastres dans cette zone importante.

Mbts-clé: Cadastremultifonction, S.I.G., gestion du risque.

\section{ABSTRACT}

Uke of themiltiparpose temitorial cadastre on riskmanagenent - at first, this workpresents a brief bib]iographical review about records and risk management. After that, the methodblogy used on the execution of thematic maps through the Intemet showing the characteristics of susceptibleluilidings to some type of risks is presented.

Finally, it denonstrates the potential andviability of using this infomation on cadastres in this important area, while at the same time using minor financial resarces.

Key words: Multipunpose cadastre, GIS, risk management.

\footnotetext{
* Commicação apresentada ao V Encontro Nacional e I Congresso Intemacional de Riscos.
} 


\section{Introdução}

O cadastro tem sido um tema bastante debatido no Brasil e no mundo. Não apenas os aspectos geométricos chamam a atenção, mas também a possibilidade de sua utilização para apoiar importantes diagnósticos que levem a propostas eficientes para soluções de problemas nas administrações municipais. Amultidisciplinaridade é a premissa básica para a implantação do cadastro Técnico Multifinalitário atualmente, diferente das discussões anteriores, nas quais era freqüente a intenção de ut i 7 izar as dadas cadastrais apenas para a tributação.

Não há como negar que a atividade de Planejamento vem ganhando espaço nas administrações municipais, dada sua importância para o desenvolvimento sustentável das municípios. Um grande passo foi dado, rumo à modernização do sistema cadastral brasileiro, no que diz respeito à integração entre cadastro e registro dos imóveis, principalmente através das exigências estabelecidas pela Lei 10267/2001 e suas regulamentações.

Um dos objetivos desta lei é acabar com as sobreposições de áreas registradas, minimizando conflitos e fortalecendb o direito de propriedade. Por outro lado, este advento fortalecerá o sistema cadastral brasileiro, principalmente no aspecto físico/geométrico (Anvrim et al, 2006) .

Cabe ressaltar que os artigas da Lei 10267/2001 não se aplicam à área urbana; as normas técnicas até então estabelecidas também se aplicam apenas à área nural. No entanto, algumas discussões já se iniciam objetivando definições e normas técnicas sobre a execução e implantação de cadastro unbano.

A questão da multidiscipl inaridade é outro fator quepode fortalecer o Cadastro TécnicoMiltifinalitário, como instrumento de planejamento, uma vez que o mesmo pode auxi liar na gestão territorial e uma das áreas que pode uti i izar estes rearrsos éa de gestão de riscos, prevenção e segurança.

A Federação Intemacional de Geâmetras (FIG) , vem estabelecendo os padrões para os sistemas cadastrais em muitos países, nos quais a multidisciplinaridade surge como fator importante para a efetivação do Cadastro Territorial Multifinalitário como instrumento de Planejamento e CestãoTerritorial.

Os Sistemas de Informações Geográficas (SIG) podem ser implantados em áreas unbanas utilizando o Banco de Dados Cadastrais. Esse sistema deve conter dadbs sobre as características constnutivas das edificações, além dos dados sociceconâmicos, que possibi i item vários tipos de análises. Undos trabalhos mais conhecidos, que utiliza as características construtivas das edificaçães é a avaliação com o dojetivo de tributação.

Atualmente, com a conscientização de que um SIG Cadastral pode se tornar um poderoso instrumento de planejamento, novas formas de util ização desses dados vem surgindo. Este trabalho apresenta o Cadastro Territorial Multifinal itário de uma cidade brasileira de pequeno porte que pode ser utilizado na gestão de riscos, prevenção e segurança a partir de aplicativos desenvolvidas com auti i ização de Softuares Livres.

\section{Revisãobibliográfica}

\section{Cacastromultifinalitário}

o Cadastro Mult ifinal itário pode ser definido como um sistema de informações territoriais, projetadb para servir tanto aas órgãos pûblicas como privadas, além de servir ao maior número passível de cidadãos, diferindo de outros sistemas territoriais por ser baseado em parcelas (Dait e McLalchin, 1990) .

Segundo Lapsson (1996), os sistemas cadastrais dos países da Europa Ocidental têm as mesmas características, pois são baseados nos princípios do sistema cadastral francês. Este consistia em uma simples descrição verbal e um mapa, no qual se detalhava a local ização e as fronteiras das terras em questão. Esta herança, deixada por Napoleão no século XIX, foi quandb da tentativa de tomar a Erropa um único Império Francês, e assim os países colonizados herdaram a semelhança desse sistema.

\section{Sistemas de Informaçães e Intemet}

De acordo com LAUDON e LAUDON (1999) "um sistema de informações pode ser definido camo um conjunto de componentes inter-relacionados que armazena, recupera, processa e apresenta informações com a finalidade de facilitar o planejamento, o controle, a análise e o processo decisório de uma empresa" .

Segundo Sthudirr e Kaufman (2002), os Sistemas de Informações Territoriais estão inseridos dentro do contexto da adninistração territorial. Dessa forma, envolvem aspectos técnicas e de recursos humanos, podendo ser considerados como sistemas de aquisição, processamento, armazenamento e disponibi l ização da informação territorial.

Embora no Brasil o termo Sistema de Informação Territorial (SIT) não seja muito difundido, emattros países ele é amplamente uti lizado, principalmente quando está integrado ao Registro de Imóveis. Em algumas localidades, o SIT é confundido com SIG, porém são distintos, se integram para auxiliar as 
processos de tomadas de decisão por parte da administração municipal.

Para que passam atingir seus dojetivos, os SIT' $\mathrm{S}$ têm sidb alvo de estudos em várias localidades, sendb necessário considerar dois aspectos importantes para que se possa tornar um SIT operacional: o estabelecimento db SIT ensi e o treinemento de rearsos humanos para que possam lidar com as novas tecnologias de organização e disponibilização das informaçães acerca do território (Wirirangav, 2002)

Como avanço da Intemet, us sistemas de informações passaram a apresentar umnovo at rativo às organizaçoues como forma de comércio eletrônico e prestação de serviços. Isso se dápelo baixo austo de desenvolvimento, implantação e manutenção desses sistemas.

Os sistemas de informaçães baseados na Intemet, em sua maioria, são compostos por web sites dinâmicos em conjunto com um banco de dados localizado no servidor remoto de arquivos. En geral, o ustário faz a requisição de informaçães através do web site, este se comunica com o banco de dados e retoma os valores em uma página de resposta.

A disponibilização de dados cadastrais na Intemet deve considerar questões relevantes como a seleção dos dados que devem compor o sistema cadastral, bem como a integridade dos mesmos. Lembrando-se ainda que nem todos os dados que compõem esse sistema podem ser disponibilizados para todos os usuários (MaJd e Willitansan, 1999; Poliey e WiLjansan, 1999).

\section{Sistemas informatizados na Administração Pỉblica}

De acordo com AlrRA-Ifurún (2005) , o planejamento de um sistema de informaçães aplicado a prefeituras deve ser similar aos desenvolvidos para empresas privadas, proporcionandb agilidade e transparência ao fluxo de informações, disponibilizando de maneira adequada as informações de caráter público e protegendb aquelas de caráter restrito.

Segundo o mesmo autor, esses sistemas facilitam a apresentação de informaçães de nível estatístico e de indicadores de gestão.

Assim, "se faz necessário um conjunto de políticas, estratégias e medidas para impulsionar a modemização dos serviços públicos por meio de sistemas informatizados" (Rosis, 2004) .

\section{Metodologia}

Pressupostos metodológicos e a inovação tecnológica em cadastro

Não é difícil apresentar uma breve abordagem sobre os pressupostos metodológicas da atividade de cadastro no Brasil, uma vez que pouco se tem avançado nesta área, mesmo com tanta evolução de métodos e técnicas proporcionados pela constante inovação tecnológica.

De acordo com Avorim et al. (2004), podem-se encontrar facilmente muitas empresas, que trabalham com levantamentos cadastrais, utilizando-se de métodos chamados convencionais totalmente arcaicos e sem uma metodologia adequada de controle de qualidade. Esses métodos, utilizados há muitos anos, são reconhecidamente onerosos e morosos, muitas vezes inviabilizando este tipo de trabalho, uma vez que a maioria das prefeituras brasileiras são pequenas e desprovidas de recursos financeiros próprios que passibil item a realização desse investimento, tão importante para a organização e o desenvolvimento municipal.

Algumas empresas procuram minimizar o trabal ho de campo e/ou de escritório, numa tentativa de reduzir custos e melhorar a qualidade dos dados. Essas iniciativas são impulsionadas, principalmente, pela existência de novas tecnologias, já existentes no mercadb, como a uti lização de coletores eletrônicos de dados, do tipo Palm Top, eliminando a fase de digitação manual dos dados coletados em campo.

Em termos de técnicas de mapeamento, notam-se nos últimos anos grandes mudanças no que diz respeito à inovação, pelo surgimento principalmente de novos instrumentos tais como GPS, imagens de satélites de alta resolução, fotografias digitais e sistemas fotogramétricos com processamentos totalmente digitais, além de uma sensível redução de custos de equipamentos e serviços, tornando o mapeamento mais acessivivel às Prefeituras.

Atualmente, algumas discussões sobre a democratização das informações cadastrais começam a surgir, no sentido de tomar o cadastro vercadeiramente multifinalitário. Un fato que auxilia significativamente a disseminação das informaçães cadastrais é a facilidade de disponibil izar as mesmas por meio da Intemet.

\section{Desenvolvimento}

O Sistema de Informações Cadastrais (SIC) foi construído cono um web site uti i izando a linguagem de programação PHP (Hypertext Preprocessor/ Préprocessadbr de hipertexto) en conjunto coma linguagem de manipulação de banco de dadbs Squ (Structured Query Language/ Linguagem de Consultas Estruturadas) .

CONHRSE e PARK (2001) descrevem o PHP como uma linguagem de criação de scripts, embutida em HIIML (Hypertext Markup Language/Linguagem de marcação dehipertexto) no servidor, quepermite adicionar funções do servidor às páginas da web tomandb-as interativas. 
Além disso, o SQL auxilia o PHP facilitando a commicação do sistema com o banco de dados.

O SQL é uma linguagem de manipulação, de informações provenientes de um banco de dados relacional que segue um padrão mundial, sendo reconhecida pela ANSI (American National Standards Institute/Instituto Nacional Americano de Padrões) e pela ISO (International Standard Organization/Organização Internacional de Padrões) (CASTAGNETTO, 2001).

Desta forma, o sistema se toma multiplataforma, uma vez que pode ser acessado como um web site em qualquer tipo de navegador web independente do sistema qperacional.

\section{Obtenção dos dados cadastrais}

Os dadas foram dbtidos através de entrevistas e medições realizadas em campo, no município de Ribeirão dos Índios - SP. Esses dados referem-se às características físicas dos imóveis, bem como à ocupação dos mesmos. Sendo assim, foram levantadas informaçães a respeito dos ocupantes do imóvel para fins de planejamento urbano, tais como dados sociceconônicos (saúde, educação, serviças, etc.) .

O levantamento cadastral urbano do município de Ribeirão das Índios - SP, foi feito uti lizandb para tanto a metodologia altemativa desenvolvida por Avorim et al. (2004), por meio de formulários que passibil itama leitura óptica de marcas. O uso dessa metodologia permite não só maior rapidez na construção do banco de dados, mas também mel hora consideravelmente o controle de qualidade dos dados, uma vez que elimina os erros de digitação por parte do operador e ainda rejeita formulários que contenham erros de preenchimento.

Esses fatos fazem com que o banco de dados seja confiável e que não haja dados incorretos. Alguns erros de integridade podem ser minimizados com essa metodologia, respeitando a modelagem previamente concebida. Neste trabalho, a modelagem do banco de dadas foi feita no software DBDesigner 4.0, usandb a abordagem entidade-relacionamento.

\section{Implementação do aplicativo}

Para a elaboração dos mapas temáticos, foram utilizadas principalmente as características das edificações que podem apresentar maior risco de incêndio e/ou desabamento, para exemplificar e avaliar a potencialidade do sistema desenvolvido. Para tanto, necessitou-se criar mapfi les com consultas real izadas diretamente no banco de dados cadastrais, ou seja, arquivos para geração de mapas baseados em conexões com o PostGIS.
O PostGIS possui um aplicativo denominado shp2pgsql, responsável por criar o código, em linguagem SQL, das informações geométricas existentes em um arquivo do tipo shape, juntamente com seus atributos. Uti i izou-se esta ferramenta para a construção do banco de dados cadastrais a part ir do material previamente disponível (shapefiles) .

Com as tabelas implementadas no sistema Postgresor, iniciou-se a elaboração de mapfi i les que se conectassem diretamente ao banco de dados para a busca de informaçães.

O I3GæO é um software para Internet baseado em um conjunto de attros softuares livres, principalmente o Mapserver. Ele apresenta um conjunto de ferramentas de navegação, compart i lhamento e geração de mapas sob demanda.

Após realizar mais pesquisas sobre este programa, verificou-se que seria uma excelente ferramenta para a implementação dos mapas cadastrais interativos de Ribeirão dos Índios na Intemet, pois alémde ser um softuare livre ainda conta com diversos aplicativos já criados em linguagem PHP, tais como o PHRMEpscript e o JavaScript.

Ao qptar pelo uso do I3GEO, necessitau-se recriar os mapfi i les conectados ao PostGIS, sendo que houve um acréscimo de alguns comandos como o MEIADATA, permitindo a configuração de permissão ou não de algumas ferramentas do I3GEO sobre determinado tema. Essas alterações são necessárias para possibi 7 itar o correto acesso dos dados inseridos nos mapfi i les, util izandb a estnutura do I3GEO.

\section{Resultadas}

Para disponibilizar a visualização de mapas temáticos, precisou-se criar uma árvore de menus, cujos temas dos layers são tratados como opções separadas em sete grupos: Educação, Saúde, Residentes, Dados Estatísticas, Dados Cadastrais, Áreas de Risco e Outros (fig. 1) .

Para este estudb, consideraram-se como grupas de Áreas de Risco os imóveis que apresentam características que os tornam susceptíveis, principalmente, ao desabamento e ao incêndio. Portanto, oprincipal mapa temático implementado foi o que representa as Imóveis com Estrutura de Madeira em estadb de conservação nuim ou regular (fig. 2) .

Os resultados apresentados procuram exempl ificar o que pode ser feito por meio do sistema implementado, mas existem muitos dadas cadastrais que podem oferecer condições de real izar vários tipos de estudos e diagnósticos, aplicáveis às mais diferentes áreas. 
territorium 16

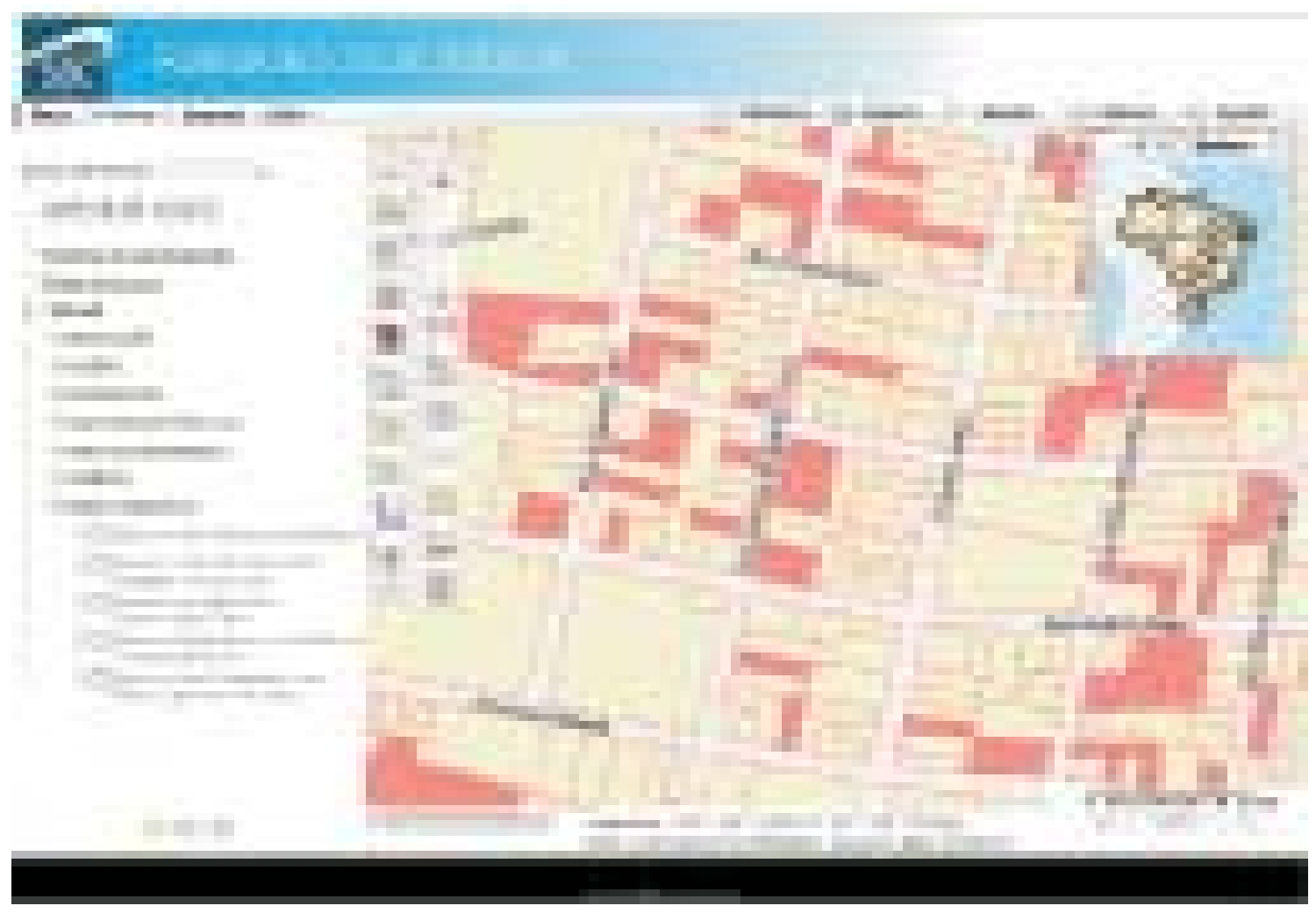

Fig. 1 - Árvore de menus.

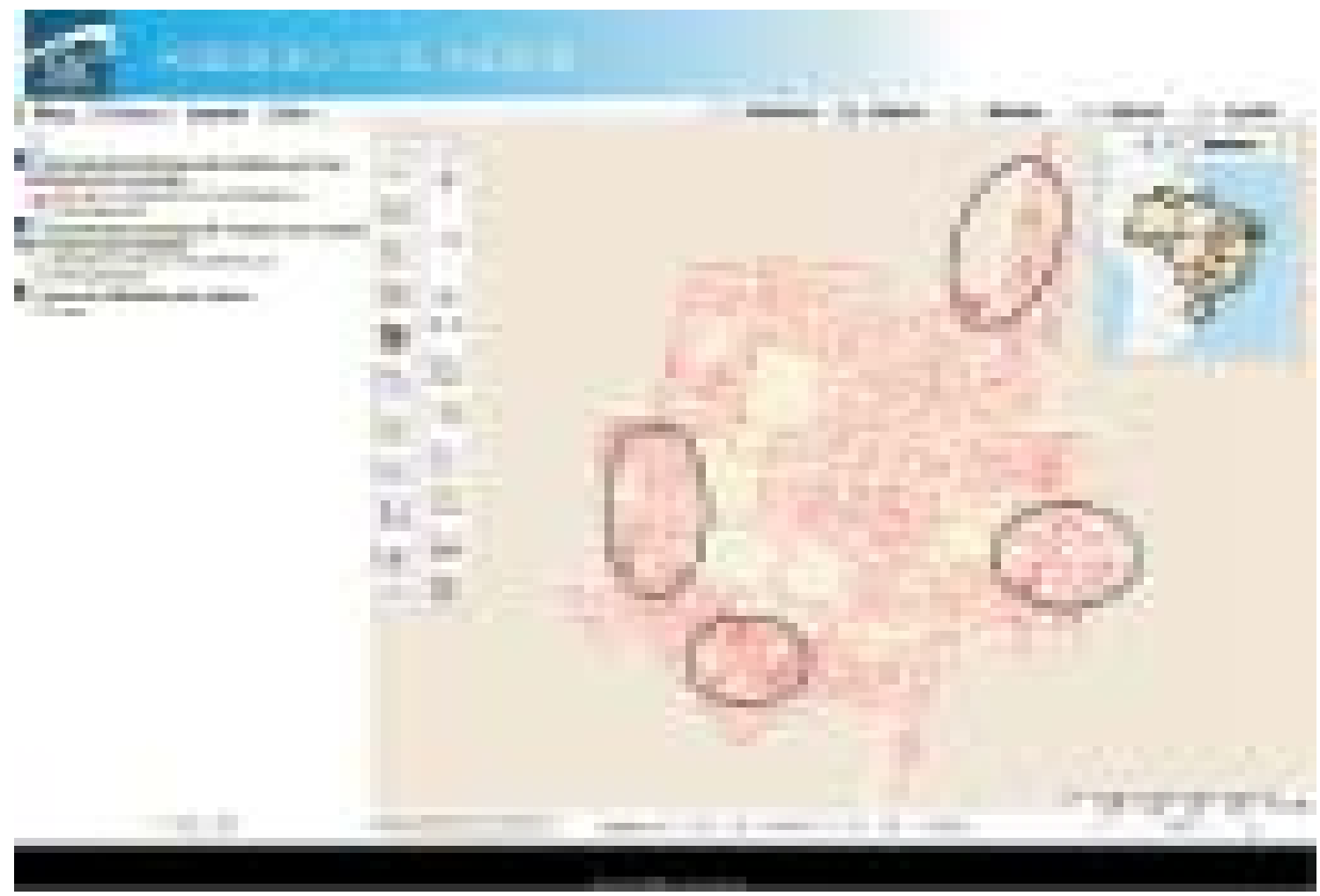

Fig. 2 - Mapa de imóveis de madeira em mau estado de conservação. 
Um exemplo interessante de aprimoramento deste mesmo experimento, seria a inclusão das imóveis nos quais residem pessoas idosas e/ou com dificuldade de locamoção. Esta informação é de grande interesse para estudos de salvamento e planejamento de ações que levem em consideração os locais com maior possibil idade de ocorrer um determinado acidente, cuja operação de salvamento pudesse ser obstruída pela dificuldade de locomoção das habitantes.

Existematros softures de SIG, inclusive livres, que oferecem ótimas ferramentas de análise que possibilitam traçar rotas para salvamento, por exemplo, que são instrumentos poderosas quando se trata de estudos e gestão de risco, mas poucos oferecem acesso direto pela Internet como neste caso.

\section{Conclusões}

A gestão de informações espaciais, nas administrações muicipais, precisa ser encarada com mais seriedade pelos governantes, uma vez que é inegável o poder da informação nos processos decisórios e a infinidade de rearsos e instnumentos de informática disponíveis atualmente à nossa disposição.

A importância da inovação tecnológica não reside apenas na possibi 7 idade de real izar traballhos com maior rapidez e menores custos, mas também na possibi l idade da ut i l ização de novos instnumentos, como a Intemet para disponibil izar informaçães com grande facilicade.

Pela proposta de reforma cadastral disautida pela FIG o cadastro analógico (em papel), com seus extensos arquivos, deverá ser extinto, dando lugar aos bancos de dados e sistemas informatizados com grande facilidade de acesso.

São inúmeras as possibilidades de estudas que podem ser real izados, uti 7 izandb os dadas constantes do cadastro territorial miltifinalitário, da forma como foi apresentada neste trabalho, que podem ser aplicadas na gestão de riscos. Para isso, basta que o projeto seja dimensionado de forma a permitir o acesso fácil e rápido às informações e que as mesmas se mantenham atualizadas.

\section{Referências bibliográficas}

Avrrm, A. et al. (2004) - "Tima metooblogia altemativa para a otimização da entrada de dados em sistemas cadastrais". Revista Brasi leira de Cartografia, ISSN 0560-4613, Rio de Janeiro, v. 56, n. 1, p. 47-54.

Anorim, A. et al. (2006) - "A modernização do Cadastro Técnico Multifinal itário Unbano e a Influência da Evolução Tecnológica: uma reflexão sobre o futuro e a multidisciplinaridade do cadastro". Anais, CDROM, COBRAC 2006. Congresso Brasileiro de Cadastro Técnico Miltifinalitário, 7, Florianq́polis.

ÄrA-Irón, A. (2005) - "Innovación en la elaboración de un Sistema de Información Geográfica (SIG) Y una Infraestnuctura de Datos Espaciales (IDE) para la gestión de la información unbanística en la administración pública" . Anais, CRROM, Semana Geomática, 6, Barcelona.

CAstagneito, J. et al. (2001) - Professional php programing. Wrox. Tradução de L. Pareschi et al. São Paulo, Makron, 770 p.

COMERsE, T; PAKk, J. (2001) - PHP4: a bílblia. Tradução de E. Frmandiewicz; J. Figueiredb. Rio de Janeiro, Campus, 697p. Titulo original: RHP4 Bible.

Date, P. F. \&MHzthiN, J. D. (1990) - Land information management, an introduction with special reference to cadastral problems in third world countries. Oxford. Oxford University Press, 259p.

Lxpsson, G. (1996) - Iand registration and cadastral systems. England, UK, Longman Group.

Lawa, C. K; Larow, J. P. (1999) - Information systems and the Intemet. Tradução deD. C. deAlencar. Rio de Janeiro, ITC.

MaJD, S. I. A. ; WiruTAvBan, I. P. (1999) - "Cadastral Systems on the World Wide Web: a Multi-purpose Vision". XXVII Anual Conference of the Australian Urban and Regional Information Systems Association. Christchurch, Nova Zelândia.

Porry, I. ; Wurtangar, I. P. (1999) - "GTS, the Intemet and the Cadastre - Coming Together" . The Australian Strveyor, Vol. 44, No 2, Austrália.

Roses, M. (2004) - Conceptos para implementar aplicaciones georeferenciadas para Intemet. Arso de Geomática, Codima, Barcelona.

Steudler, D. ; Kaufvann, J. (2002) - Benchmarking Cadastral Systems, FIG, Dermark, 77 p.

Wurfanban, I. P. (2002) - "The Cadastral "Tool Box" A Framework for Reform" . Anais, CDROM, FIG Intemational Congress, 22. Washington, DC, EHA. 